\title{
Providing probability distributions for the causal pathogen of clinical mastitis using naive Bayesian networks
}

\author{
W. Steeneveld, ${ }^{* 1}$ L. C. van der Gaag, $†$ H. W. Barkema,‡ and H. Hogeveen* $\S$ \\ ${ }^{*}$ Department of Farm Animal Health, Faculty of Veterinary Medicine, Utrecht University, PO Box 80151, 3508 TD Utrecht, the Netherlands \\ †Department of Information and Computing Sciences, Utrecht University, PO Box 80089, 3508 TB Utrecht, the Netherlands \\ $\ddagger$ Department of Production Animal Health, Faculty of Veterinary Medicine, University of Calgary, Calgary, T2N 4N1 Canada \\ §Chair group Business Economics, Wageningen University, PO Box 8130, 6706 KN Wageningen, the Netherlands
}

\begin{abstract}
Clinical mastitis $(\mathrm{CM})$ can be caused by a wide variety of pathogens and farmers must start treatment before the actual causal pathogen is known. By providing a probability distribution for the causal pathogen, naive Bayesian networks (NBN) can serve as a management tool for farmers to decide which treatment to use. The advantage of providing a probability distribution for the causal pathogen, rather than only providing the most likely causal pathogen, is that the uncertainty involved is visible and a more informed treatment decision can be made. The objective of this study was to illustrate provision of probability distributions for the gram status and for the causal pathogen for $\mathrm{CM}$ cases. For constructing the NBN, data were used from 274 Dutch dairy herds in which the occurrence of CM was recorded over an 18-mo period. The data set contained information on $3,833 \mathrm{CM}$ cases. Two-thirds of the data set was used for the construction process and one-third was retained for validation. One NBN was constructed with the CM cases classified according to their gram status, and another was built with the CM cases classified into streptococci, Staphylococcus aureus, or Escherichia coli. Information usually available at a dairy farm was included in both NBN (parity, month in lactation, season of the year, quarter position, SCC and CM history, being sick or not, and color and texture of the milk). Accuracy was calculated to obtain insight in the quality of the constructed NBN. The accuracy of classifying CM cases into gram-positive or gram-negative pathogens was $73 \%$, while the accuracy of classifying CM cases into streptococci, Staph. aureus, or E. coli was $52 \%$. Because only CM cases with a high probability for a single causal pathogen will be consid-
\end{abstract}

Received September 9, 2008.

Accepted January 19, 2009.

${ }^{1}$ Corresponding author: w.steeneveld@uu.nl ered for pathogen-specific treatment, accuracies based on only classifying CM cases above a particular probability threshold were determined. For instance, for CM cases in which either gram-negative or gram-positive had a probability $>0.90$, classification according to the gram status reached an accuracy of $97 \%$. We found that the greater the probability for a particular pathogen was for a CM case, the more accurate was the classification of this case as being caused by this pathogen. The probability distributions provided by the NBN and the associated accuracies for varying classification thresholds provide the farmer with considerable insight about the most likely causal pathogen for a CM case and the uncertainty involved.

Key words: clinical mastitis, diagnosis, pathogen, dairy

\section{INTRODUCTION}

Mastitis is one of the most frequent and costly diseases in a dairy herd (e.g., Halasa et al., 2007). A large proportion of the total cost of mastitis is caused by clinical mastitis (CM; Huijps et al., 2008). Effective treatment of CM is important to eliminate and prevent recurrence of IMI and to identify effective methods of control based on limited or no use of antibiotics to reduce the likelihood of resistance (Hillerton and Kliem, 2002).

Knowing the causal pathogen and subsequently using appropriate treatment serves to increase the cure rate of CM (Barkema et al., 2006; McDougall et al., 2007). Although bacteriological culturing will provide information about the causal pathogen, this information typically comes too late, because CM needs treatment immediately after diagnosis. In practice therefore, a choice of treatment needs to be made in the absence of culture results. Several other sources of information are available on a dairy farm that could aid in indicating the causal pathogen of a CM case to some extent. Particular pathogens more frequently cause $\mathrm{CM}$ in some seasons of the year than in other seasons (Makovec 
and Ruegg, 2003; Olde Riekerink et al., 2007). Also, cow-specific factors such as parity, DIM, test-day SCC, and CM history are a valuable source of information (Zadoks et al., 2001; de Haas et al., 2004). Finally, clinical signs such as the appearance of the milk and the demeanor of the cow can be used to aid in indicating the causal pathogen (Jones and Ward, 1990; Milne et al., 2003).

In previous studies, various classification models for pathogen identification for $\mathrm{CM}$ were constructed. However, the predictive performance of these models varied strongly (e.g., Jones and Ward, 1990; Kim and Heald, 1999; Milne et al., 2003). A further disadvantage of these models was that they only returned the most likely causal pathogen to a farmer. For choosing among treatment options, a probability distribution for the causal pathogens of a CM case would be more informative as it reveals the uncertainty involved in the classification. For instance, almost equal probabilities for 2 or more causal pathogens would support the decision for a broad-spectrum antibiotic treatment whereas a very high probability for a particular pathogen would support the choice for a more specific treatment. As an example, for gram-negative CM cases (Escherichia coli and Klebsiella spp.), supportive treatment is more appropriate than antimicrobial treatment (Morin et al., 1998; Pyörälä and Pyörälä, 1998).

Hogeveen et al. (1994) suggested the use of Bayesian networks for the task of pathogen identification for CM. A Bayesian network includes a collection of stochastic variables representing sources of information and can generate posterior probability distributions for any outcome variable of interest. Naive Bayesian networks (NBN) are the simplest type of Bayesian network. These networks are well known for their powerful performance on classification tasks (Friedman et al., 1997). Naive Bayesian networks have been studied extensively and are being widely applied in human medicine (e.g., Kukar et al., 1999; Blanco et al., 2005; Chapman et al., 2005). Applications are still relatively rare in veterinary medicine, but they are gaining in popularity (McKendrick et al., 2000; Geenen et al., 2005; Kuncheva et al., 2007).

The objective of this study was to illustrate the value of providing probability distributions for the gram status and for the causal pathogen for $\mathrm{CM}$ cases to a farmer to make a more informed treatment decision. Naive Bayesian networks were used for computing casespecific probability distributions. These networks were created automatically from data. A data set of 3,833 CM cases was used with information about the causal pathogen and associated information on clinical signs, cow factors, and season.

\section{MATERIALS AND METHODS}

\section{Available Data}

The data available for the present study were described in detail elsewhere (Barkema et al., 1998). In short, data on CM were collected from 274 dairy herds entering the study between December 1992 and June 1994; each herd participated for approximately $1.5 \mathrm{yr}$. All herds had an annual milk production quota between 300,000 and $900,000 \mathrm{~kg}$, and had cows of the HolsteinFriesian or Dutch Friesian breeds. The herd size had a mean of 75 cows and ranged from 40 to 143 cows. Lactating cows were housed in a free-stall barn during winter and were milked in a double-herringbone or 2-sided tandem parlor. During the study, farmers were asked to collect milk samples from cows with signs of CM. The samples were taken before treatment, stored in a freezer at the farm (at approximately $-20^{\circ} \mathrm{C}$ ), and collected every 6 to $8 \mathrm{wk}$ for bacteriological culturing. For each $\mathrm{CM}$ case, the farmer further provided information on cow identification, date of occurrence, infected quarter, and whether the cow was sick at the moment of CM. In the laboratory, bacteriological culturing of the milk samples was performed according to the standards of the National Mastitis Council (Harmon et al., 1990), and the causal pathogen was determined. Furthermore, the texture and color of the milk were scored independently by 2 technicians after thawing and before bacteriological culturing (Barkema et al., 1998). The Dutch national milk recording system (Nederlands Rundvee Syndicaat, Arnhem, the Netherlands) provided information from the 3- or 4-weekly milk production recording, including cow identification, date of milk recording, date of calving, date of drying off, test-day milk yields ( $\mathrm{kg}$ of milk, fat, and protein), and SCC (cells $/ \mathrm{mL})$ for all cows in the study.

\section{Data Preparation}

The originally available data set consisted of 8,571 CM cases. To ensure that no unrecorded previous cases of CM had occurred within the lactation, only $\mathrm{CM}$ cases from lactations that had been recorded from calving onward were eligible for inclusion. From these, $\mathrm{CM}$ cases from lactations without any milk production information, or with a calving interval $\leq 320 \mathrm{~d}$ or $\geq 600$ d were excluded. Clinical mastitis cases during dry-off were also excluded and intervals between pathogen-specific cases of $\mathrm{CM}$ in the same quarter had to be at least $14 \mathrm{~d}$ for a case to be included in the final data set. All contaminated samples, culture-negative samples, and $\mathrm{CM}$ cases where no sample was taken were excluded. 
Table 1. Number of clinical mastitis cases per data set

\begin{tabular}{|c|c|c|c|}
\hline Item & Cases, $\mathrm{n}$ & Training, $\mathrm{n}$ & Validation, $\mathrm{n}$ \\
\hline \multicolumn{4}{|c|}{ Data sets 1 and $1 b^{1}$} \\
\hline Gram-negative ${ }^{2}$ & 1.006 & 685 & 321 \\
\hline Gram-positive $^{3}$ & 2,528 & 1,647 & 881 \\
\hline Total & 3,534 & 2,332 & 1,202 \\
\hline \multicolumn{4}{|l|}{ Data sets 2 and $2 b^{4}$} \\
\hline STREP $^{5}$ & 962 & 647 & 315 \\
\hline STAPH $^{6}$ & 746 & 459 & 287 \\
\hline $\mathrm{COLI}^{7}$ & 979 & 661 & 318 \\
\hline Total & 2,687 & 1,767 & 920 \\
\hline \multicolumn{4}{|c|}{$\begin{array}{l}{ }^{1} \text { Data set } 1 \text { contained gram-specific clinical mastitis history, data set } 1 \mathrm{~b} \text { contained information on whether or } \\
\text { not the cow had clinical mastitis before the current clinical mastitis in the same lactation. }\end{array}$} \\
\hline \multicolumn{4}{|c|}{${ }^{2}$ Escherichia coli $(\mathrm{n}=923)$, Klebsiella $(\mathrm{n}=56)$, Pseudomonas $(\mathrm{n}=17)$, and mixed cultures $(\mathrm{n}=10)$. } \\
\hline \multicolumn{4}{|c|}{$\begin{array}{l}{ }^{3} \text { Streptococcus dysgalactiae }(\mathrm{n}=369) \text {, Streptococcus agalactiae }(\mathrm{n}=21) \text {, Streptococcus uberis }(\mathrm{n}=254) \text {, } \\
\text { other streptococci }(\mathrm{n}=288) \text {, Staphylococcus aureus }(\mathrm{n}=746) \text {, coagulase-negative staphylococci }(\mathrm{n}=215) \text {, } \\
\text { Arcanobacterium pyogenes }(\mathrm{n}=27) \text {, Corynebacterium bovis }(\mathrm{n}=90) \text {, and mixed cultures }(\mathrm{n}=518) .\end{array}$} \\
\hline \multicolumn{4}{|c|}{$\begin{array}{l}{ }^{4} \text { Data set } 2 \text { contained pathogen-specific clinical mastitis history, data set } 2 \mathrm{~b} \text { contained information on whether } \\
\text { or not the cow had clinical mastitis before the current clinical mastitis in the same lactation. }\end{array}$} \\
\hline \multirow{2}{*}{\multicolumn{4}{|c|}{$\begin{array}{l}{ }^{5} \text { Strep. dysgalactiae }(\mathrm{n}=369) \text {, Strep. agalactiae }(\mathrm{n}=21) \text {, Strep. uberis }(\mathrm{n}=254) \text {, other streptococci }(\mathrm{n}=288) \text {, } \\
\text { and mixed cultures of streptococci }(\mathrm{n}=30) \text {. }\end{array}$}} \\
\hline & & & \\
\hline \multicolumn{4}{|c|}{${ }^{7}$ E. $\operatorname{coli}(\mathrm{n}=923)$ and Klebsiella $(\mathrm{n}=56)$. } \\
\hline
\end{tabular}

The final data set for our study now consisted of 3,833 CM cases of 3,018 cows in 3,581 different quarters.

From the final data set, 2 different enhanced versions were constructed (Table 1): data set 1 included an additional variable classifying each $\mathrm{CM}$ case according to the causal pathogen's gram status (GRAM), and data set 2 included an additional variable classifying each CM case according to the causal pathogen (PATH). In data set 1, all CM cases were classified as either grampositive CM (Streptococcus dysgalactiae, Streptococcus agalactiae, Streptococcus uberis, other streptococci, Staphylococcus aureus, CNS) or gram-negative CM (E. coli, Pseudomonas, and Klebsiella). Mixed cultures containing 2 gram-positive pathogens were classified as gram-positive and those containing 2 gram-negative pathogens as gram-negative. All CM cases that could not be classified according to their gram status were excluded from data set $1(\mathrm{n}=299)$. In data set 2 , all CM cases were classified into STREP, containing all streptococci (Strep. dysgalactiae, Strep. agalactiae, Strep. uberis, and other streptococci), STAPH, containing Staph. aureus, and COLI, containing E. coli and Klebsiella. Mixed cultures containing pathogens from the same class were classified into this class (e.g., a mixed culture containing Strep. uberis and Strep. dysgalactiae was classified as STREP). All CM cases that could not be classified into STREP, STAPH, or COLI $(\mathrm{n}=1,146)$ were excluded from data set 2 .

Risk and indicator variables for the gram status in data set 1 and for the pathogen group in data set 2 were defined using information from the literature and based on the expertise of the authors (Table 2). In both data sets, the parity, month in lactation, location of infected quarter, and season of the year were available for each CM case. Three variables on SCC were constructed (e.g., de Haas et al., 2004). If available, the test-day SCC 1 to $30 \mathrm{~d}$ before CM (SCC1) and the closest test-day SCC $>30 \mathrm{~d}$ before CM (SCC2) were determined. Additionally, for multiparous cows, geometric mean SCC from all available test-day records from the previous lactation (PrevSCC) was defined. Because continuous variables cannot be handled in standard Bayesian networks, the values of all SCC variables were classified as either $<$ or $\geq 200,000$ cells $/ \mathrm{mL}$ (Dohoo and Leslie, 1991). For both data sets, moreover, pathogen-specific CM history variables were defined (Zadoks et al., 2001). For data set 1 , these variables were defined as the gram status of the causal pathogen 1 to $30 \mathrm{~d}$ and $>30 \mathrm{~d}$ before the current CM. For data set 2, these variables were defined as the pathogen itself 1 to $30 \mathrm{~d}$ and $>30 \mathrm{~d}$ before the current CM. In view of the projected use of the developed models, however, it is noted that pathogenspecific CM history information often is not available on farms but only when milk samples of previous CM cases have been collected. Therefore, 2 additional data sets were created, containing the same cases as data sets 1 and 2, respectively, but instead of the variables on pathogen-specific CM history, non-pathogen-specific variables were defined. These variables were defined as whether the cow had CM or not in the previous $30 \mathrm{~d}$ and $>30 \mathrm{~d}$ ago in the current lactation. These data sets will be referred to as data set $1 \mathrm{~b}$ and data set $2 \mathrm{~b}$.

Because hardly any $\mathrm{CM}$ information from previous lactations was available in the data sets, no such infor- 
Table 2. Description of the study variables with their abbreviation and different levels used for the construction of naive Bayesian networks

\begin{tabular}{|c|c|c|c|}
\hline Description & Abbreviation & Classes, $\mathrm{n}$ & Classifications \\
\hline Pathogen of current CM case & PATH & 3 & STREP, ${ }^{2}$ STAPH $^{3}{ }^{\mathrm{COLI}^{4}}$ \\
\hline Parity & PAR & 4 & $1,2,3, \geq 4$ \\
\hline Season of the year & SEAS & 4 & $\begin{array}{l}\text { January-March, April-June, July-September, } \\
\text { October-December }\end{array}$ \\
\hline Quarter position of the udder & $\mathrm{QP}$ & 4 & Right front, left front, right rear, left rear \\
\hline SCC $>30$ d before current CM & SCC2 & 2 & $\begin{array}{l}<200,000 \text { cells } / \mathrm{mL} \\
\geq 200,000 \text { cells } / \mathrm{mL}\end{array}$ \\
\hline Geometric mean SCC in previous lactation & PrevSCC & 2 & $\begin{array}{l}<200,000 \text { cells } / \mathrm{mL} \\
\geq 200,000 \text { cells } / \mathrm{mL}\end{array}$ \\
\hline CM history $1-30 \mathrm{~d}$ before current $\mathrm{CM}$ & CM1 & 2 & No, yes \\
\hline CM history $>30 \mathrm{~d}$ before current $\mathrm{CM}$ & CM2 & 2 & No, yes \\
\hline Gram history 1-30 d before current CM & GRAM1 & 3 & No previous CM, gram-positive, gram-negative \\
\hline Cow sick at moment of $\mathrm{CM}$ & SICK & 2 & Not sick, sick \\
\hline
\end{tabular}

${ }^{1} \mathrm{CM}=$ clinical mastitis.

${ }^{2}$ Streptococcus dysgalactiae, Streptococcus agalactiae, Streptococcus uberis, other streptococci and mixed cultures of streptococci.

${ }^{3}$ Staphylococcus aureus.

${ }^{4}$ Escherichia coli and Klebsiella.

mation was taken into account in our study. There were missing values for the variables on SCC1 (26\%), SCC2 $(40 \%)$ and PrevSCC (22\%). In all data sets, finally, 3 variables were included with information on the clinical signs (Jones and Ward, 1990; Milne et al., 2003): color of the milk, texture of the milk, and whether or not the cow was sick. These variables had $<1 \%$ missing values each.

\section{Model Building}

An NBN consists of a single class variable that represents the possible classes for the dependent variable, and a set of feature variables modeling the relevant levels of the independent variables. In data sets 1 and $1 \mathrm{~b}$, the variable GRAM was the class variable; in data sets 2 and $2 \mathrm{~b}$, the variable PATH was the class variable. All other variables in the 4 data sets were potential feature variables. From each of the 4 data sets, an NBN was constructed, resulting in a total of 4 models. These NBN differed in their class variable and in the feature variables on $\mathrm{CM}$ history. For the models $\mathrm{NBN}_{\mathrm{GRAM} 1}$ and $\mathrm{NBN}_{\text {GRAM1b}}$, GRAM was taken as the class variable. For constructing $\mathrm{NBN}_{\mathrm{GRAM} 1}$, the feature variables modeling gram-specific history (GRAM1 and GRAM2) were used; while for constructing $\mathrm{NBN}_{\mathrm{GRAM1b}}$, the less specific feature variables capturing CM history (CM1 and CM2) were taken into consideration. Similarly, for
$\mathrm{NBN}_{\text {PATH2 }}$ and $\mathrm{NBN}_{\text {PATH2b }}$, the class variable was PATH; for $\mathrm{NBN}_{\mathrm{PATH} 2}$, the feature variable modeling pathogenspecific history (PATH1 and PATH2) were used and for $\mathrm{NBN}_{\text {PATH2b}}$, the less specific feature variables with CM history (CM1 and CM2).

Naive Bayesian networks are typically constructed from data that consists of determining prior probabilities for the class variable and of estimating conditional probabilities for the feature variables given the possible classes of the dependent variable. In this study, the prior probabilities for the class variables reflect the prevalence of gram-positive pathogens and gram-negative pathogens among the CM cases (gram-positive, $70.6 \%$; gram-negative, $29.4 \%$ ), and the prevalence of the 3 pathogen groups (STREP, $36.6 \%$; STAPH, $26.0 \%$; and COLI, $37.4 \%$ ) in the data sets used for construction (see also Table 1). The conditional probabilities for the feature variables are based on frequency counts in the data. For instance, the conditional probability of a cow being sick, given that it is infected with a gramnegative pathogen; that is, the probability $\operatorname{Pr}($ Sick $=$ yes $\mid$ gram status = negative), was computed as the proportion of sick cows among the CM cases reporting a gram-negative pathogen.

In essence, all available feature variables can be included in an NBN. Methods exist, however, for selecting only those feature variables that best discriminate between the different classes of a dependent variable, 
thereby forestalling overfitting of the data (Langley and Sage, 1994). For this study, a wrapper method with forward selection was used for selecting appropriate feature variables (e.g., Blanco et al., 2005; Geenen et al., 2005). With this method, feature variables were selected to optimize the accuracy of the NBN under construction. The method started with an NBN including just the class variable and no feature variables. In each subsequent step, it computed the accuracy of the NBN with a single feature variable added, for each such variable separately. It then included the feature variable that increased the accuracy the most, if any. The inclusion of feature variables was continued until the accuracy of the NBN no longer improved.

For the purpose of constructing and subsequently validating the $4 \mathrm{NBN}$, the data sets were split into a training set and a validation set. From each data set, two-thirds of the herds were selected randomly for training; the $\mathrm{CM}$ cases of the remaining herds were included in a validation data set (Table 1). Selecting herds rather than separate CM cases was aimed at applicability of the resulting models on farms. The selection process apparently did not result in a bias, as the prevalence of pathogens was approximately equal in the training and validation data sets (Table 1). Construction of the NBN was done with 10 -fold cross validation on the training data set. The NBN that performed best on the training set during cross validation was selected for further evaluation on the validation data set. Constructing the different NBN, which includes estimating the prior and conditional probabilities, was done by using the Bayesian-network editing package Dazzle (Schrage et al., 2005).

\section{Validation}

Obtaining Posterior Probabilities. The 4 constructed NBN were used to calculate posterior probability distributions for the class variables (GRAM and PATH), given information on the selected feature variables, for each CM case from the validation data set. For computing posterior probabilities, an NBN builds upon Bayes's rule together with the assumption that all feature variables are mutually independent given the class variable. More specifically, for computing the posterior probability $\operatorname{Pr}\left(c \mid f_{1}, \ldots, f_{n}\right)$ of the class $c$ given levels $f_{1}, \ldots, f_{n}$ for its $n$ feature variables, the model uses

$$
\operatorname{Pr}\left(c \mid f_{1}, \ldots, f_{n}\right)=\frac{\prod_{i=1}^{n} \operatorname{Pr}\left(f_{i} \mid c\right)^{*} \operatorname{Pr}(c)}{\sum_{j=1}^{k} \prod_{i=1}^{n} \operatorname{Pr}\left(f_{i} \mid c_{j}\right)^{*} \operatorname{Pr}\left(c_{j}\right)}
$$

where $c_{1}, \ldots, c_{k}$ are the possible classes for the model's class variable. For the class variable GRAM, for example, $c_{1}$ equals the class of gram-negative pathogens and $c_{2}$ equals the class of gram-positive pathogens. For the class variable PATH, $c_{1}$ equals STREP, $c_{2}$ equals $\mathrm{STAPH}$, and $c_{3}$ equals COLI. In the formula, $\operatorname{Pr}(c)$ is the prior probability of the CM being caused by the group $c$ of pathogens. $\operatorname{Pr}\left(f_{i} \mid c_{j}\right)$ are the conditional probabilities of the level $f_{i}$ of the $i$ th selected feature variable given that the CM is caused by the group $c_{j}$ of pathogens. Note that the prior probabilities $\operatorname{Pr}(c)$ and the conditional probabilities $\operatorname{Pr}\left(f_{i} \mid c_{j}\right)$ for all $i$ and $j$, have already been estimated from the data upon constructing the NBN and therefore are readily available in the NBN for the computation of the posterior probabilities using formula [1]. The Dazzle package could have been used for computing the posterior probabilities for the gram status and for the causal pathogen of a CM case but we decided to use Microsoft Excel. For this purpose, a log-odds transform of formula [1] was used, which resulted in a simple additive formula, which in turn could be easily implemented in Microsoft Excel.

Model Accuracy. From the posterior probability distribution computed for the class variable for a CM case, the predicted class was established. For the GRAM variable, which had 2 possible classes, the predicted class was the gram-positive class if its posterior probability was $>0.71$; otherwise, the predicted class was gram-negative. The threshold probability of 0.71 was chosen to reflect the prevalence of gram-positive $\mathrm{CM}$ cases in the data set. For the PATH class variable, having 3 possible classes, the predicted class was the one with highest posterior probability. If 2 or more classes had equal posterior probabilities, ties were broken at random. Subsequently, the predicted class was compared with the known causal pathogen of the case. By performing these computations for each $\mathrm{CM}$ case from the validation data set, the accuracy of the model was established, that is, the percentage of correctly classified cases.

In practice on a farm, only CM cases with a very high posterior probability for a single pathogen or group of pathogens will be eligible for pathogen-specific treatment; CM cases that resulted in more or less equal posterior probabilities will be more eligible for broadspectrum use of antibiotics. By providing probability distributions for the causal pathogens or groups of pathogens, the farmer can readily distinguish between such cases. To establish the accuracy of the models for CM cases with a high posterior probability (for instance, $>0.80$ ) for a single class, all other cases were left unclassified. As an example, suppose a data set consisting of 
Table 3. Overview of the order in which the feature variables were included in the final naive Bayesian networks ${ }^{1}$

\begin{tabular}{lllll}
\hline Order & NBN $_{\mathrm{GRAM} 1}{ }^{2}$ & $\mathrm{NBN}_{\mathrm{GRAM} 1 \mathrm{~b}}{ }^{3}$ & $\mathrm{NBN}_{\mathrm{PATH} 2}{ }^{4}$ & $\mathrm{NBN}_{\mathrm{PATH} 2 \mathrm{~b}}{ }^{5}$ \\
\hline First added feature & SICK & SICK & SCC1 & SCC1 \\
Second added feature & GRAM1 & COLOR & PATH1 & SICK \\
Third added feature & COLOR & SCC1 & SICK & TEXT \\
Fourth added feature & GRAM2 & TEXT & PATH2 & SEAS \\
Fifth added feature & SCC1 & SCC2 & SCC2 & CM2 \\
Sixth added feature & - & - & TEXT & - \\
Seventh added feature & - & - & SEAS & - \\
Eighth added feature & - & COLOR & - \\
\hline
\end{tabular}

${ }^{1} \mathrm{SEAS}=$ season of the year; SCC1 $=$ SCC 1 to $30 \mathrm{~d}$ before current clinical mastitis $(\mathrm{CM})$; SCC $2=\mathrm{SCC}>30 \mathrm{~d}$ before current CM; CM2 = CM history $>30 \mathrm{~d}$ before current CM; PATH1 = pathogen history 1 to $30 \mathrm{~d}$ before current $\mathrm{CM}$; PATH2 = pathogen history $>30 \mathrm{~d}$ before current CM; GRAM1 = gram history 1 to $30 \mathrm{~d}$ before current CM; GRAM2 = gram history $>30 \mathrm{~d}$ before current CM; COLOR = color of the milk of cow with CM; TEXT $=$ texture of the milk of cow with CM; SICK = whether cow was sick at moment of CM.

${ }^{2}$ Naive Bayesian network including gram-specific clinical mastitis history.

${ }^{3}$ Naive Bayesian network including whether or not cow had clinical mastitis before the current clinical mastitis in the same lactation.

${ }^{4}$ Naive Bayesian network including pathogen-specific clinical mastitis history.

${ }^{5}$ Naive Bayesian network including whether or not cow had clinical mastitis before the current clinical mastitis in the same lactation.

1,000 CM cases, 400 of which had a posterior probability for their gram status $>0.80$; the remaining 600 CM cases had posterior probabilities $<0.80$. The 400 CM cases were then used for determining the model's accuracy. If 325 of these cases were classified correctly, for instance, the accuracy of the model for CM cases with probabilities $>0.80$ would be $81 \%$. These accuracies will be called stratified accuracies, because they are based upon different strata of the data set under study. All accuracies were calculated using SAS, version 9.1 (SAS Institute Inc., Cary, NC).

\section{RESULTS}

\section{Naive Bayesian Networks}

A total of $4 \mathrm{NBN}$ were constructed from the 4 data sets. After feature selection, $\mathrm{NBN}_{\text {GRAM1 }}$ and $\mathrm{NBN}_{\text {GRAM1b }}$ contained 5 feature variables, $\mathrm{NBN}_{\mathrm{PATH} 2}$ contained 8 feature variables, and $\mathrm{NBN}_{\text {РАтн2b }}$ contained 5 feature variables (Table 3). Upon feature selection for $\mathrm{NBN}_{\text {GRAM1 }}$ and $\mathrm{NBN}_{\text {GRAM1b }}$, the feature variable SICK, modeling whether or not the cow was sick at the moment of the current $\mathrm{CM}$, increased the accuracy the most and was selected first. In addition, the feature variable COLOR, capturing the color of the cow's milk, proved to be a relatively strong indicator for the gram status of the current $\mathrm{CM}$. For $\mathrm{NBN}_{\mathrm{PATH} 2}$ and $\mathrm{NBN}_{\mathrm{PATH} 2 \mathrm{~b}}$, the feature variable SCC1 was selected first. Also, the feature variable SICK had an ability to discriminate between the possible causal pathogens for the current CM. For $\mathrm{NBN}_{\text {GRAM1 }}$, the feature variables capturing gram-specific CM history information (GRAM1 and GRAM2) were selected (Table 3). Similarly, the feature variables modeling pathogen-specific CM history information (PATH1 and PATH2) were included in $\mathrm{NBN}_{\mathrm{PATH} 2}$ quite early in the feature selection process.

\section{Validation}

Obtaining Posterior Probabilities. For each CM case from the validation data sets, posterior probability distributions were established using the constructed NBN. For instance, a sick cow with CM, with watery milk in the sample, with an SCC in the last month $<200,000$ cells/mL, and in this lactation no CM before the current $\mathrm{CM}$, had a posterior probability of a gramnegative pathogen causing the CM of 0.74 and a posterior probability of a gram-positive pathogen causing the $\mathrm{CM}$ of 0.26 . A cow with $\mathrm{CM}$, which was not sick, with a normal color of the milk, with an SCC in the last month $\geq 200,000$ cells $/ \mathrm{mL}$, and in this lactation no CM before the current CM, had a posterior probability of a gram-negative pathogen of 0.33 and a posterior probability of a gram-positive pathogen of 0.67 .

Another example was a cow with CM in April, which was sick, with watery milk and small flakes in the milk, with 2 previous SCC measurements $\geq 200,000$ cells $/ \mathrm{mL}$. For this lactation, moreover, there was no CM before the current CM. There was a posterior probability for STREP, STAPH, and COLI of 0.38, 0.24, and 0.38, respectively. Another cow experienced CM in January, was not sick, had milk with a very yellow color and with big flakes, experienced 2 previous SCC measurements $<200,000$ cells $/ \mathrm{mL}$, and had no CM before the current $\mathrm{CM}$ in this lactation. The posterior probability 
Table 4. Predicted and actual numbers (\%) of clinical mastitis cases for gram-negative and gram-positive pathogens for $\mathrm{NBN}_{\mathrm{GRAM} 1}{ }^{1}$

\begin{tabular}{lccr}
\hline & \multicolumn{2}{c}{ Actual } \\
\cline { 2 - 3 } Prediction & Gram-negative & Gram-positive & \multicolumn{1}{c}{ Total } \\
\hline Gram-negative & $197(49)$ & $204(51)$ & $401(100)$ \\
Gram-positive & $124(15)$ & $677(85)$ & $801(100)$ \\
Total & 321 & 881 & 1,202
\end{tabular}

${ }^{1}$ Naive Bayesian network including gram-specific clinical mastitis history.

for STREP, STAPH, and COLI was 0.15, 0.06, and 0.79 , respectively.

Model Accuracy. Using a threshold probability of 0.71 for predicting a $\mathrm{CM}$ case as being gram-positive resulted in accuracies of $73 \%$ and $70 \%$ for $\mathrm{NBN}_{\text {GRAM1 }}$ and $\mathrm{NBN}_{\mathrm{GRAM} 1 \mathrm{~b}}$, respectively. The difference in accuracy between the 2 gram-predicting models was not significant $(P=0.15)$. Table 4 presents, for the $\mathrm{NBN}_{\text {GRAM1 }}$ model, the predicted and actual numbers of cases for each gram status. Out of the 1,202 CM cases in the validation data set, a total of 874 (i.e., $197+$ 677 ) cases were classified correctly by the NBN, which resulted in an accuracy of $73 \%$ (i.e., 874/1,202). Of the 801 gram-positive predicted CM cases, 677 cases were indeed caused by gram-positive pathogens; $85 \%$ of the gram-positive predicted cases were thus classified correctly. Of the 401 gram-negative predicted CM cases, 197 cases were caused by gram-negative pathogens; $49 \%$ of the gram-negative predicted cases were thus classified correctly.

Using the most likely pathogen as the predicted value, the PATH-predicting models gave accuracies of 52 and $48 \%$ for $\mathrm{NBN}_{\text {PATH2 }}$ and $\mathrm{NBN}_{\text {PATH2b }}$, respectively. The difference in accuracy was significant $(P=0.05)$. Table 5 presents the predicted and actual numbers of cases for each group of causal pathogens for the $\mathrm{NBN}_{\mathrm{PATH} 2}$ model. Out of the $920 \mathrm{CM}$ cases in the validation data set, a total of 480 (i.e., $144+126+210$ ) cases were classified correctly by the NBN, which resulted in an accuracy of $52 \%$ (i.e., 480/920). Of the 305 STREP-predicted CM cases, 144 cases (i.e., 47\%) were indeed caused by STREP. The NBN further classified $31 \%$ of these 305 STREP-predicted cases incorrectly as STAPH and another $22 \%$ as COLI. Of the total of $228 \mathrm{CM}$ cases with STAPH as the predicted causal pathogen, 126 cases (i.e., 55\%) were indeed caused by STAPH. However, $27 \%$ of the 228 cases were incorrectly predicted as STREP and another $18 \%$ were predicted as COLI. For the $387 \mathrm{CM}$ cases predicted as COLI, the percentage of correctly classified cases was $54 \% ; 29 \%$ of the 387 cases were predicted as STREP and the remaining $17 \%$ were predicted as STAPH.
Table 5. Predicted and actual numbers (\%) of clinical mastitis cases for each group of pathogens using the most likely pathogen as the predicted value for $\mathrm{NBN}_{\mathrm{PATH} 2}{ }^{1}$

\begin{tabular}{lrrrr}
\hline & \multicolumn{3}{c}{ Actual } & \\
\cline { 2 - 4 } Prediction & \multicolumn{1}{c}{ STREP $^{2}$} & \multicolumn{1}{c}{ STAPH $^{3}$} & \multicolumn{1}{c}{ COLI $^{4}$} & Total \\
\hline STREP $^{2}$ & $144(47)$ & $94(31)$ & $67(22)$ & $305(100)$ \\
STAPH $^{3}$ & $61(27)$ & $126(55)$ & $41(18)$ & $228(100)$ \\
COLI $^{4}$ & $110(29)$ & $67(17)$ & $210(54)$ & $387(100)$ \\
Total & 315 & 287 & 318 & 920
\end{tabular}

${ }^{1}$ Naive Bayesian network including pathogen-specific clinical mastitis history.

${ }^{2}$ Streptococcus dysgalactiae, Streptococcus agalactiae, Streptococcus uberis, other streptococci and mixed cultures of streptococci.

${ }^{3}$ Staphylococcus aureus.

${ }^{4}$ Escherichia coli and Klebsiella.

Stratified accuracies were computed for the GRAMpredicting models (Table 6) and for the PATH-predicting models (Table 7), using different strata. For instance, with the $\mathrm{NBN}_{\text {GRAM1 }}$ model, a total of $601 \mathrm{CM}$ cases (equal to $50 \%$ of the $1202 \mathrm{CM}$ cases in the validation data set) resulted in a posterior probability of over 0.80 of the CM being caused by a gram-positive pathogen or, alternatively, by a gram-negative pathogen. Of these $601 \mathrm{CM}$ cases, $90 \%$ were classified correctly. With the same model, a total of $186 \mathrm{CM}$ cases resulted in a posterior probability of over 0.95 for one of the 2 possible classes of the gram status of the causal pathogen. All these $186 \mathrm{CM}$ cases were classified correctly, resulting in a stratified accuracy of $100 \%$. Table 8 details the stratified accuracy of the $\mathrm{NBN}_{\text {GRAM1 }}$ model for cases with a posterior probability exceeding a threshold value of 0.90 . Out of the $342 \mathrm{CM}$ cases in the data set for which a posterior probability of over 0.90 was computed for a gram-negative pathogen or, alternatively, for a grampositive pathogen, a total of 330 (i.e., $309+21$ ) cases were classified correctly by the NBN, which resulted in a stratified accuracy of $97 \%$ (i.e., 330/342). Of the 321 gram-positive predicted CM cases, 309 cases (i.e., 96\%) were indeed caused by a gram-positive pathogen. All of the 21 gram-negative predicted CM cases were indeed caused by a gram-negative pathogen.

Using $\mathrm{NBN}_{\text {PATH2 }}$ with pathogen-specific CM history information, $155 \mathrm{CM}$ cases resulted in a posterior probability distribution in which STREP, STAPH, or COLI had a posterior probability of over 0.80 . Of these 155 CM cases, $76 \%$ were classified correctly. Table 9 details the stratified accuracy of the $\mathrm{NBN}_{\mathrm{PATH} 2}$ model for cases with a posterior probability exceeding a threshold value of 0.90 . Out of the $84 \mathrm{CM}$ cases in the data set for which a posterior probability of over 0.90 was computed for one of the causal pathogens, a total of 70 (i.e., $1+23$ +46 ) cases were classified correctly by the NBN, which 
Table 6. Stratified accuracy at different thresholds on the posterior probability of the most likely gram status for $\mathrm{NBN}_{\mathrm{GRAM} 1}$ and $\mathrm{NBN}_{\text {GRAM1b }}$

\begin{tabular}{|c|c|c|c|c|}
\hline \multirow{2}{*}{$\begin{array}{l}\text { Threshold } \\
\text { on posterior } \\
\text { probability }\end{array}$} & \multicolumn{2}{|c|}{$\mathrm{NBN}_{\text {GRAM1 }}{ }^{1}$} & \multicolumn{2}{|c|}{$\mathrm{NBN}_{\text {GRAM1b }}{ }^{2}$} \\
\hline & $\begin{array}{l}\text { Number }(\%) \text { of } \mathrm{CM} \\
\text { cases above threshold }\end{array}$ & $\begin{array}{c}\text { Stratified } \\
\text { accuracy }(\%)\end{array}$ & $\begin{array}{l}\text { Number }(\%) \text { of } \mathrm{CM} \\
\text { cases above threshold }\end{array}$ & $\begin{array}{c}\text { Stratified } \\
\text { accuracy }(\%)\end{array}$ \\
\hline 0.70 & $911(76)$ & 83 & $900(75)$ & 81 \\
\hline 0.75 & $798(66)$ & 86 & $817(68)$ & 83 \\
\hline 0.80 & $601(50)$ & 90 & $660(55)$ & 86 \\
\hline 0.85 & $433(36)$ & 94 & $538(45)$ & 89 \\
\hline 0.90 & $342(28)$ & 97 & $378(31)$ & 90 \\
\hline 0.95 & $186(15)$ & 100 & $168(14)$ & 96 \\
\hline
\end{tabular}

${ }^{1}$ Naive Bayesian network including gram-specific clinical mastitis (CM) history.

${ }^{2}$ Naive Bayesian network including whether or not cow had clinical mastitis before the current clinical mastitis in the same lactation.

amounts to a stratified accuracy of $83 \%$ (i.e., 70/84). The STREP-predicted CM case was indeed caused by STREP. Of the 29 STAPH-predicted CM cases, $79 \%$ were caused by STAPH; $17 \%$ of the 29 cases, however, were incorrectly predicted as STREP and the remaining $4 \%$ were predicted as COLI. Of the 54 COLI-predicted CM cases, $85 \%$ were caused by COLI; $9 \%$ of the 54 cases, however, were incorrectly predicted as STREP and the remaining $6 \%$ were predicted as STAPH.

\section{DISCUSSION}

Bayesian networks are known to be powerful tools for knowledge representation and probabilistic inference (Jensen, 2001). The networks are flexible in terms of capturing complex dependencies among their variables and allow, in essence, the computation of any prior or posterior probability of interest for the modeled variables. These characteristics favor Bayesian networks in comparison with other statistical methods such as logistic regression, for probabilistic reasoning tasks. Even NBN, the simplest type of Bayesian network, are surprisingly effective (Langley and Sage, 1994). Naive Bayesian networks build upon the assumption that all feature variables are mutually independent given the class variable and provide for computing a (posterior) probability distribution for the class variable. This probability distribution then is used for classification purposes. As reported by Friedman et al. (1997), the classification performance of NBN is good, even if the independence assumption for the feature variables does not hold true in the data. Relaxing the independence assumption could, in essence, lead to more accurate classification models. In tree-augmented NBN (Friedman et al., 1997), for instance, a tree-like dependency structure over the feature variable is constructed: each feature variable then is directly dependent of the class variable and of at most one other feature variable. Creating tree-augmented NBN, however, includes searching for dependencies between feature variables. For accurately recovering these dependencies from a data set, more data need to be available than for building an NBN. A tree-augmented NBN is often a good compromise between an NBN and a Bayesian network based

Table 7. Stratified accuracy at different thresholds on the posterior probability of the most likely causal pathogen for $\mathrm{NBN}_{\mathrm{PATH} 2}$ and $\mathrm{NBN}_{\mathrm{PATH} 2 \mathrm{~b}}$

\begin{tabular}{|c|c|c|c|c|}
\hline \multirow{2}{*}{$\begin{array}{l}\text { Threshold } \\
\text { on posterior } \\
\text { probability }\end{array}$} & \multicolumn{2}{|c|}{$\mathrm{NBN}_{\text {PATH2 }}{ }^{1}$} & \multicolumn{2}{|c|}{$\mathrm{NBN}_{\text {PATH } 2 \mathrm{~b}}{ }^{2}$} \\
\hline & $\begin{array}{l}\text { Number }(\%) \text { of } \mathrm{CM} \\
\text { cases above threshold }\end{array}$ & $\begin{array}{c}\text { Stratified } \\
\text { accuracy }(\%)\end{array}$ & $\begin{array}{l}\text { Number (\%) of } \mathrm{CM} \\
\text { cases above threshold }\end{array}$ & $\begin{array}{c}\text { Stratified } \\
\text { accuracy }(\%)\end{array}$ \\
\hline 0.70 & $258(28)$ & 73 & $112(12)$ & 73 \\
\hline 0.75 & $207(23)$ & 77 & $89(10)$ & 78 \\
\hline 0.80 & $155(17)$ & 76 & $54(6)$ & 83 \\
\hline 0.85 & $115(13)$ & 81 & $30(3)$ & 97 \\
\hline 0.90 & $84(9)$ & 83 & $16(2)$ & 100 \\
\hline 0.95 & $36(4)$ & 89 & $9(1)$ & 100 \\
\hline
\end{tabular}

${ }^{1}$ Naive Bayesian network including pathogen-specific clinical mastitis (CM) history.

${ }^{2}$ Naive Bayesian network including whether or not cow had clinical mastitis before the current clinical mastitis in the same lactation. 
Table 8. Predicted and actual numbers (\%) of clinical mastitis cases for gram-negative and gram-positive pathogens with a posterior probability over 0.90 for $\mathrm{NBN}_{\mathrm{GRAM} 1}{ }^{1}$

\begin{tabular}{lccc}
\hline & \multicolumn{2}{c}{ Actual } \\
\cline { 2 - 3 } Prediction & Gram-negative & Gram-positive & Total \\
\hline Gram-negative & $21(100)$ & $0(0)$ & $21(100)$ \\
Gram-positive & $12(4)$ & $309(96)$ & $321(100)$ \\
Total & 33 & 309 & 342 \\
\hline
\end{tabular}

${ }^{1}$ Naive Bayesian network including gram-specific clinical mastitis history.

on causality; the model structure is simple enough to avoid overfitting, but strong dependencies can be taken into account.

In the present study, in addition to the $4 \mathrm{NBN}$, tree-augmented NBN were also constructed (data not shown). These tree-augmented networks had lower accuracies than their matching NBN. An explanation of these lower accuracies is that the relatively small data sets available in our study caused unreliable estimates upon searching for dependencies and led to an irrelevant dependence structure (Friedman and Goldszmidt, 1996). Another explanation is that the feature variables in our study did not contain enough information to make a clear distinction between the causal pathogens and that the constructed tree-augmented NBN, as a consequence of their larger number of estimates, tend to overfit the data. For these reasons, we chose to use NBN even though their lack of dependencies between the feature variables is somewhat unrealistic. Although some of the modeled independencies are likely to hold, it is questionable, for example, whether the color and the texture of the milk of a cow with CM are independent given the gram status of the causal pathogen.

The constructed NBN can be used to generate a posterior probability distribution of the gram status of a $\mathrm{CM}$ case or on the causal pathogen. Based upon this distribution, the farmer can make an informed treatment decision. The gram-positive pathogens Strep. uberis and Staph. aureus, for example, need different treatment regimens; the duration of the treatment strongly influences the cure rate (Sol et al., 2000; Barkema et al., 2006). Because of the expected low cure rate for Staph. aureus (e.g., Sol et al., 2000; Taponen et al., 2003; McDougall et al., 2007), moreover, a decision to cull the cow instead of treating the cow should also be considered. Posterior probabilities of STREP, STAPH, and COLI, as provided by the NBN, give the farmer insight into the likelihood of Staph. aureus compared with, for example, Strep. uberis, allowing him to carefully evaluate and weigh the possible consequences of different treatment regimens.
For the different NBN, the feature variables SICK and SCC1 were selected first, indicating that these variables served to increase the accuracy the most. Whenever pathogen-specific CM history variables were available in the data sets, these variables were selected for the NBN, which indicates that these variables have some predictive value for the causal pathogen. In contrast, the feature variables on non-pathogen-specific CM history (CM1 and CM2) were included late or not at all in the feature selection process, which indicates that these variables do not contain enough information for distinguishing between the different groups of pathogens. In all NBN, at least one of the feature variables modeling the color and the texture of the milk was selected. The importance of these variables for pathogen diagnosis of $\mathrm{CM}$ cases was also reported in previous studies (e.g., Jones and Ward, 1990; Zadoks et al., 2001, de Haas et al., 2004).

The main purpose of this study was to illustrate the value of providing probability distributions for the gram status and for the causal pathogen to allow a farmer to make a more informed treatment decision. A validation of a probability distribution is not possible. It is still valuable, however, to gain at least some insight into the quality of the constructed NBN. For this purpose, the accuracy was determined for all constructed NBN. The accuracy was also used as an indication of the model's quality in other studies of NBN (e.g., Blanco et al., 2005; Geenen et al., 2005) and in studies on other classification models for CM (e.g., Kim and Heald, 1999; Heald et al., 2000; Milne et al., 2003). In other studies classifying CM cases according to their gram status (Jones and Ward, 1990; Milne et al., 2003) or into E. coli or not (White et al., 1986a, b), accuracy varied between 71 and $79 \%$. There are no related studies reporting accuracies for classifying CM causal pathogens into STREP, STAPH, or COLI.

Table 9. Predicted and actual number (\%) of clinical mastitis cases for each group of pathogens with a posterior probability over 0.90 for $\mathrm{NBN}_{\text {PATH2 }} 1$

\begin{tabular}{lcccc}
\hline & \multicolumn{3}{c}{ Actual } \\
\cline { 2 - 4 } Prediction & STREP $^{2}$ & STAPH $^{3}$ & COLI $^{4}$ & Total \\
\hline STREP $^{2}$ & $1(100)$ & $0(0)$ & $0(0)$ & $1(100)$ \\
STAPH $^{3}$ & $5(17)$ & $23(79)$ & $1(4)$ & $29(100)$ \\
COLI $^{4}$ & $5(9)$ & $3(6)$ & $46(85)$ & $54(100)$ \\
Total & 11 & 26 & 47 & 84 \\
\hline
\end{tabular}

${ }^{1}$ Naive Bayesian network including pathogen-specific clinical mastitis history.

${ }^{2}$ Streptococcus dysgalactiae, Streptococcus agalactiae, Streptococcus uberis, other streptococci and mixed cultures of streptococci.

${ }^{3}$ Staphylococcus aureus.

${ }^{4}$ Escherichia coli and Klebsiella. 
It was also reported that trained veterinarians reached an accuracy of $62 \%$ in classifying CM cases into E. coli or not (White et al., 1986b). Because of the different prevalences of the causal pathogens in these studies, the reported accuracies are not readily comparable with the accuracies obtained in the present study. In the present study, the accuracies computed for the $\mathrm{NBN}_{\mathrm{GRAM} 1}$ and $\mathrm{NBN}_{\text {GRAM1b }}$ models were 73 and $70 \%$, respectively. For the purpose of comparison, note that a simple model using random guessing based upon prevalence would have an accuracy of $58 \%$; a model that attributes each CM case to a gram-positive pathogen, in turn, would have an accuracy of $70 \%$. These observations suggest that the feature variables included in the data sets are not indicative enough to make a marked distinction between gram-positive and gram-negative pathogens. Stratified accuracies were established to gain some insight in the reliability of high posterior probabilities for a single pathogen. Table 6 shows that the stratified accuracies of the gram-predicting models increase with greater threshold probabilities and, in fact, reach very high values. For $28 \%$ of the CM cases, for instance, a stratified accuracy of $97 \%$ was reached. These high stratified accuracies indicate that the greater a posterior probability computed for either a positive or a negative gram status of the causal pathogen, the more the farmer can rely on the classification result. For a CM case with a high probability for the gram status, a more specific treatment can thus be decided upon. The results of the exact predictions for CM cases with posterior probabilities over 0.90 indicate that high posterior probabilities on both gram-positive and gram-negative pathogens are reliable (Table 7).

The accuracies computed for the $\mathrm{NBN}_{\mathrm{PATH} 2}$ and NB$\mathrm{N}_{\text {PATH2b }}$ models were 52 and $48 \%$, respectively. Because the 3 groups of causal pathogens occur in approximately equal numbers in the data set, these accuracies clearly improve on random guessing, which would result in an accuracy of approximately $30 \%$. Also, a model that attributes each CM case to a single fixed pathogen (e.g., COLI) would have an accuracy of approximately $30 \%$. These observations suggest that the feature variables included in the data sets carry more information for distinguishing between the different groups of pathogens. Moreover, classifying CM cases into STREP, STAPH, or COLI resulted in high stratified accuracies. For instance, for $4 \%$ of the CM cases a stratified accuracy of $89 \%$ was reached. The NBN models with feature variables on pathogen-specific CM history had significantly higher accuracies than the NBN models with feature variables on non-pathogen-specific CM history.

The present study was based on the observation that simply classifying CM cases according to their gram status or their causal pathogen (i.e., simply present- ing the most likely value) does not provide sufficient information to assist a farmer in deciding on the most appropriate treatment for CM. With that method, only the most likely pathogen is presented to a farmer. In our study, classifying (as the accuracy does) was only used to determine the overall performance of the NBN. In our opinion, a probability distribution for the causal pathogen, revealing the uncertainty involved, provides more information for deciding upon the most appropriate treatment for CM. The advantage of providing such probability distributions is that the end-users could interpret the uncertainty involved and make a more informed treatment decision. For instance, consider a CM case with STREP for the predicted class, and assume that the posterior probability distribution for the causal pathogens equals $0.45,0.15$, and 0.40 for STREP, STAPH, and COLI, respectively. Providing only the predicted class would ignore the high posterior probability for COLI, which could cause seriously wrong treatment decisions. In practice, only CM cases for which a single pathogen receives a high posterior probability will be eligible for more specific treatment rather than broad spectrum use of antibiotics.

Because training and validation of the $4 \mathrm{NBN}$ was performed on data coming from different farms, results of this study can be generalized to all farms. In the present study, data from herds with different pathogen prevalences were combined in a single data set and analyzed together. It is likely that performance of the presented NBN can be improved by performing separate analyses on different groups of farms according to their pathogen prevalences, as was done by Heald et al. (2000), provided that sufficient data are available for each group. The class variable in the NBN, however, captures the prior probabilities on the causal groups of pathogens (GRAM or PATH) and can be adapted easily to farm-specific pathogen prevalences. For instance, for farms with a known high proportion of $E$. coli cases and consequentially a high prior probability on this pathogen, using farm-specific pathogen prevalences would result in greater posterior probabilities for COLI. The prior probabilities on the causal groups of pathogens can, moreover, be adapted continually; that is, new evidence on the distribution of pathogens as a result of bacteriological culturing of CM cases can be included directly in the NBN, and posterior probabilities will more reliably reflect reality on the farm.

The NBN used in this study can be included in automated decision support systems that support the choice and duration of treatment for each CM case separately. Because most information sources used in this study are available on most dairy farms, such systems can be installed at the farm site and used by the farmer. Using the NBN would not include extra costs, because the 
information is readily available. However, for almost all $\mathrm{CM}$ cases there will remain some uncertainty about the causal group of pathogens. Wrong treatment decisions will therefore still be made, with economic losses as a consequence. For instance, treating a cow against gram-negative pathogens when it was infected with gram-positive pathogens may result in high economic losses caused by decreased milk production and infection of other cows. In future work, therefore, economic consequences of the different treatment options will be included in the models to provide optimal advice on the choice of treatment.

\section{CONCLUSIONS}

Using NBN, a probability distribution for the gram status and for the causal group of pathogens can be given for each CM case. To obtain insight into the quality of the constructed NBN, the accuracy was determined. The accuracy of classifying CM cases into gram-positive or gram-negative pathogens was $73 \%$, whereas the accuracy of classifying CM cases into STREP, STAPH, or COLI was $52 \%$. Because only CM cases with a high probability on a single causal pathogen will be considered for pathogen-specific treatment, accuracies based on only classifying CM cases above a particular probability threshold were determined as well. For CM cases with high posterior probabilities, a reliable prediction for the causal pathogen can be given. Using the provided probability distribution for the causal pathogen, the farmer can interpret the uncertainty involved and can make a more informed decision. On a farm, this information can result in an improvement in comparison with the current situation where information on the causal pathogen is not available. The probability distributions were based on information sources which are usually available at a farm. This makes it possible to use results of this study in automated decision support systems to aid decision on choice and duration of treatment for each individual CM case.

\section{ACKNOWLEDGMENTS}

This research was supported by the Dutch Technology Foundation STW, applied science division of NWO, and the Technology Program of the Dutch Ministry of Economic Affairs.

\section{REFERENCES}

Barkema, H. W., Y. H. Schukken, T. J. G. M. Lam, M. L. Beiboer, H Wilmink, G. Benedictus, and A. Brand. 1998. Incidence of clinical mastitis in dairy herds grouped in three categories by bulk milk somatic cell counts. J. Dairy Sci. 81:411-419.
Barkema, H. W., Y. H. Schukken, and R. N. Zadoks. 2006. Invited review: The role of cow, pathogen, and treatment regimen in the therapeutic success of bovine Staphylococcus aureus mastitis. J. Dairy Sci. 89:1877-1895.

Blanco, R., M. Inza, M. Merino, J. Quiroga, and P. Larrañaga. 2005. Feature selection in Bayesian classifiers for the prognosis of survival of cirrhotic patients treated with TIPS. J. Biomed. Inform. 38:376-388.

Chapman, W. W., J. N. Dowling, and M. M. Wagner. 2005. Classification of emergency department chief complaints into 7 syndromes: A retrospective analysis of 527.228 patients. Ann. Emerg. Med. 46:445-455.

de Haas, Y., R. F. Veerkamp, H. W. Barkema, Y. T. Gröhn, and Y. H. Schukken. 2004. Associations between pathogen-specific cases of clinical mastitis and somatic cell count patterns. J. Dairy Sci. $87: 95-105$.

Dohoo, I. R., and K. E. Leslie. 1991. Evaluation of changes in somaticcell counts as indicators of new intramammary infections. Prev. Vet. Med. 10:225-237.

Friedman, N., D. Geiger, and M. Goldszmidt. 1997. Bayesian network classifiers. Mach. Learn. 29:131-163.

Friedman, N., and M. Goldszmidt. 1996. Building classifiers using Bayesian networks. Pages 1277-1284 in Proc. Natl. Conf. Artificial Intelligence, Menlo Park, CA.

Geenen, P. L., L. C. Van der Gaag, W. L. A. Loeffen, and A. R. W. Elbers. 2005. Naive Bayesian classifiers for the clinical diagnosis of classical swine fever. Pages 169-176 in Proc. $2005 \mathrm{Mtg}$. Soc. Veterinary Epidemiology and Preventive Medicine. Nairn, Inverness, Scotland.

Halasa, T., K. Huijps, O. Østerås, and H. Hogeveen. 2007. Economic effects of bovine mastitis and mastitis management: A review. Vet. Q. 29:18-31.

Harmon, R. J., R. J. Eberhart, D. E. Jasper, B. E. Langlois, and R A. Wilson. 1990. Microbiological Procedures for the Diagnosis of Bovine Udder Infection. Natl. Mastitis Counc., Inc., Arlington, VA

Heald, C. W., T. Kim, W. M. Sischo, J. B. Cooper, and D. R Wolfgang. 2000. A computerized mastitis decision aid using farmbased records: An artificial neural network approach. J. Dairy Sci. $83: 711-720$

Hillerton, J. E., and K. E. Kliem. 2002. Effective treatment of Streptococcus uberis clinical mastitis to minimize the use of antibiotics. J. Dairy Sci. 85:1009-1014.

Hogeveen, H., M. A. Varner, D. S. Bree, D. E. Dill, E. N. NoordhuizenStassen, and A. Brand. 1994. Knowledge representation methods for dairy decision-support systems. J. Dairy Sci. 77:3704-3715.

Huijps, K., T. J. G. M. Lam, and H. Hogeveen. 2008. Costs of mastitis: Facts and perception. J. Dairy Res. 75:113-120.

Jensen, F. V. 2001. Bayesian Networks and Decision Graphs. SpringerVerlag, New York, NY.

Jones, G. F., and G. E. Ward. 1990. Evaluation of a scheme for predicting the gram-staining reaction of organisms causing bovine mastitis. J. Am. Vet. Med. Assoc. 196:597-599.

Kim, T., and C. W. Heald. 1999. Inducing inference rules for the classification of bovine mastitis. Comput. Electron. Agric. 23:2742 .

Kukar, M., I. Kononenko, C. Groselj, K. Kralj, and J. Fettich. 1999. Analysing and improving the diagnosis of ischaemic heart disease with machine learning. Artif. Intell. Med. 16:25-50.

Kuncheva, L. I., V. J. D. Vilas, and J. J. Rodriguez. 2007. Diagnosing scrapie in sheep: A classification experiment. Comput. Biol. Med. 37:1194-1202.

Langley, P., and S. Sage. 1994. Induction of selective Bayesian classifiers. Pages 399-406 in Proc. 10th Conf. Uncertainty in Artificial Intelligence. Morgan Kaufmann Publishers, San Francisco, CA.

Makovec, J. A., and P. L. Ruegg. 2003. Results of milk samples submitted for microbiological examination in Wisconsin from 1994 to 2001. J. Dairy Sci. 86:3466-3472.

McDougall, S., K. E. Agnew, R. Cursons, X. X. Hou, and C. R. W Compton. 2007. Parenteral treatment of clinical mastitis with 
tylosin base or penethamate hydriodide in dairy cattle. J. Dairy Sci. 90:779-789.

McKendrick, I. J., G. Gettinby, Y. Gu, S. W. J. Reid, and C. W. Revie. 2000. Using a Bayesian belief network to aid differential diagnosis of tropical bovine diseases. Prev. Vet. Med. 47:141-156.

Milne, M. H., A. M. Biggs, J. L. Fitzpatrick, G. T. Innocent, and D. C. Barrett. 2003. Use of clinical information to predict the characteristics of bacteria isolated from clinical cases of bovine mastitis. Vet. Rec. 152:615-617.

Morin, D. E., P. D. Constable, and G. C. McCoy. 1998. Use of clinical parameters for differentiation of gram-positive and gram-negative mastitis in dairy cows vaccinated against lipopolysaccharide core antigens. J. Am. Vet. Med. Assoc. 212:1423-1431.

Olde Riekerink, R. G. M., H. W. Barkema, and H. Stryhn. 2007. The effect of season on somatic cell count and the incidence of clinical mastitis. J. Dairy Sci. 90:1704-1715.

Pyörälä, S. H. K., and E. O. Pyörälä. 1998. Efficacy of parenteral administration of three antimicrobial agents in treatment of clinical mastitis in lactating cows: 487 cases (1989-1995). J. Am. Vet. Med. Assoc. 212:407-412.

Schrage, M. M., and A. van Ijzendoorn, and L. C. van der Gaag. 2005. Haskell ready to Dazzle the real world. Pages 17-26 in Proc. 2005 ACM SIGPLAN Workshop on Haskell.
Sol, J., O. C. Sampimon, H. W. Barkema, and Y. H. Schukken. 2000. Factors associated with cure after therapy of clinical mastitis caused by Staphylococcus aureus. J. Dairy Sci. 83:278-284.

Taponen, S., K. Dredge, B. Henriksson, A. M. Pyyhtia, L. Suojala, R. Junni, K. Heinonen, and S. Pyörälä. 2003. Efficacy of intramammary treatment with procaine penicillin $G$ vs. procaine penicillin $\mathrm{G}$ plus neomycin in bovine clinical mastitis caused by penicillin-susceptible, gram-positive bacteria-A double blind field study. J. Vet. Pharmacol. Ther. 26:193-198.

White, M. E., L. T. Glickman, F. D. Barnes-Pallesen, E. G. Pearson, M. E. Montgomery, D. Armstrong, R. P. Wickenden, and G. Hickey. 1986a. Discriminant analysis of the clinical indicants for bovine coliform mastitis. Cornell Vet. 76:335-341.

White, M. E., L. T. Glickman, F. D. Barnes-Pallesen, E. S. Stem III, P. Dinsmore, M. S. Powers, P. Powers, M. C. Smith, M. E. Montgomery, and D. Jasko. 1986b. Accuracy of a discriminant analysis model for prediction of coliform mastitis in dairy cows and a comparison with clinical prediction. Cornell Vet. 76:342-347.

Zadoks, R. N., H. G. Allore, H. W. Barkema, O. C. Sampimon, G. J. Wellenberg, Y. T. Gröhn, and Y. H. Schukken. 2001. Cow- and quarter-level risk factors for Streptococcus uberis and Staphylococcus aureus mastitis. J. Dairy Sci. 84:2649-2663. 